(C2007 IEEE. Personal use of this material is permitted. However, permission to reprint/republish this material for advertising or promotional purposes or for creating new collective works for resale or redistribution to servers or lists, or to reuse any copyrighted component of this work in other works must be obtained from the IEEE. 


\title{
High Frequency Transformer Computer Modeling
}

\author{
Ahmed A. Siada, and Syed Islam, Senior Membe, r IEEE
}

\begin{abstract}
Frequency Response Analysis (FRA) technique is nowadays widely used in both factory and field applications as a diagnostic tool for power transformers. The main problem about FRA techniques is to interpret the observed evolution of the frequency response in order to identify failures. Transformer high frequency computer modeling is proposed in this work to be used with the FRA. The physical meaning of the model parameters allows the identification of the problem inside the transformer. The paper presents the model and experimental results and analysis of the frequency responses on the variations of the winding parameters. Effect of core, bushings, oil and tank on FRA is also investigated.
\end{abstract}

\section{INTRODUCTION}

$\mathrm{P}$ wer transformers are a vital link in a power system. Monitoring and diagnostic techniques are essential to decrease maintenance and improve reliability of the equipment. Currently there are several of chemical and electrical diagnostic techniques applied for power transformers[1]. Frequency response analysis (FRA) has been successfully used for detecting winding deformation, core and clamping structure [2-4]. Deformation results in relative change to the internal inductance and capacitance of the winding structure. These changes can be detected externally by FRA method. FRA is an offline test and is used to measure the input/output relationship as a function of frequency (typically in the range of $2 \mathrm{MHz}$ ). This provides a fingerprint of a transformer and is compared with its previous signatures to detect winding displacement. However, the fingerprints are rarely available, especially for transformers in service. Thus other information such as comparison between identically constructed transformers has to be taken for diagnosis. For linear systems, the system transfer function is independent of the applied signal and it only depends on the system parameters. A transformer represents a nonlinear system and its electrical equivalent circuit is very complex network of distributed resistive, capacitive, inductive elements and conductance between high voltage and low voltage windings. Hence, the frequency response of a transformer is dependent on the formulation of complex series and parallel resonant circuits. FRA with experience based interpretation identifies different problems associated with certain frequency bands.
Frequencies scan less than $5 \mathrm{kHz}$ are sensitive to core deformation, open circuits, shorted turns and residual magnetism. The frequencies scan between $10 \mathrm{kHz}$ and 500 $\mathrm{kHz}$ are sensitive mainly to bulk winding movements. High frequencies scan greater than $750 \mathrm{kHz}$ are sensitive to movement of inter-connections, winding leads, tap-changer connection and if at very high spectrum greater than $1 \mathrm{MHz}$ then even to the external connection of the test cables[5]. Conventional FRA has been relying on a graphical analysis for transformer diagnosis, which requires trained experts to interpret test results and identify failures. This paper investigates the FRA in details and presents a general model for the transformer in a broad band of frequencies. The physical meaning of the model parameters allows the identification of the problem inside the transformer.

\section{XPERIMENTAL RESULTS}

The FRA was carried out on a 15 MVA $22 / 0.415 \mathrm{kV}$ power transformer (vector group dY11). The test conditions were oil winding temperature $25^{\circ} \mathrm{C}$, ambient temperature $22^{\circ} \mathrm{C}$ and relative humidity $46 \%$. The FRA graph for high and low voltage windings is shown in Figures 1 and 2 respectively. It can be shown from Fig. 1 that in the low frequency range (less than $1 \mathrm{kHz}$ ) the response is characterized by resonance at 400 and $500 \mathrm{~Hz}$ these resonant frequencies corresponding to the half-wave space harmonics in the winding due to the low impedance terminations of the measuring circuit. Below $1 \mathrm{kHz}$ the transformer winding response is dominated by inductance. As the frequency increases more space harmonics are built up in the winding. In the medium frequency range multiple resonances can be observed over the entire frequency range. 


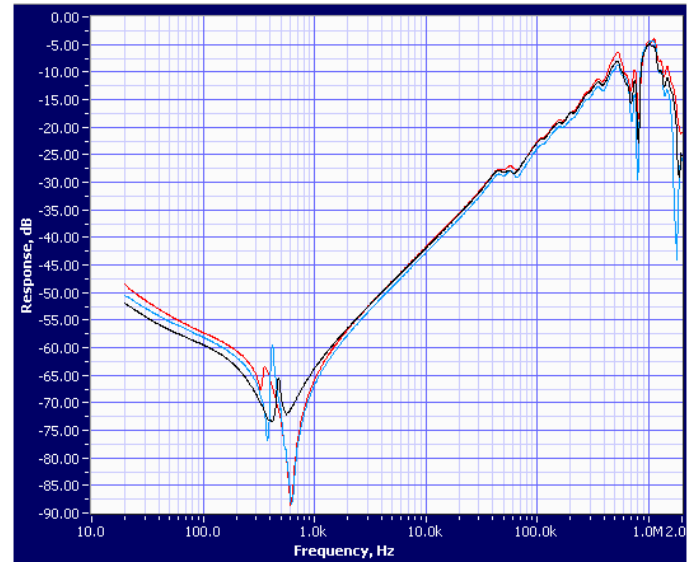

Fig. 1 High Voltage Windings

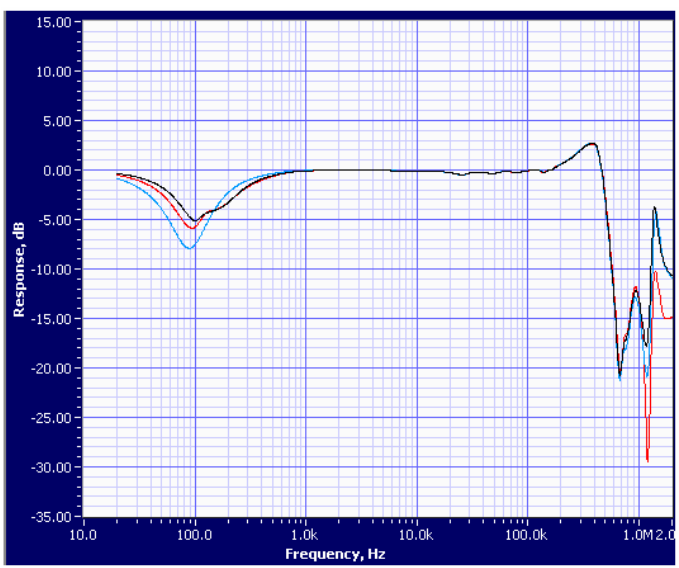

Fig. 2 Low Voltage Windings

At higher frequencies the distributed capacitances in the transformer tends to shunt the winding inductance and resonance is much less pronounced. Also winding lead effects tend to affect the results in the higher frequency region. The core will likely have some effect at the lower frequencies and skin effect will become a factor at higher frequencies.

Figure 3 shows the response when the low voltage windings are short circuited, the resonant frequencies will appear only in the higher frequency range, while the response in the very low frequency range showing some variation between the blue phase and other two phases. The distortion appears in the high frequency range is most probably caused by the end leads associated with tap-changer leads and bushing tails.

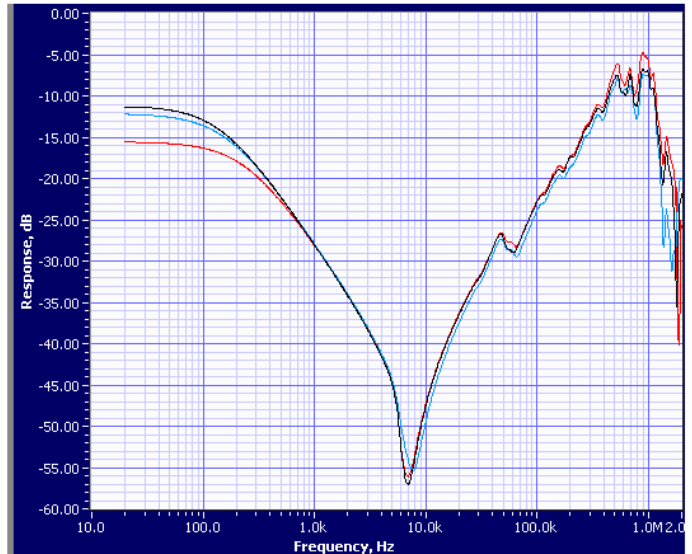

Fig. 3 FRA of HV windings with LV winding shorted

To show the effect of the core on the FRA, the windings have been removed from the transformer i.e. bushings; core, oil and tank are removed. Figures 4 and 5 show the FRA of the $\mathrm{HV}$ and LV winding respectively. The overall response is noticeably altered than those of Figures 1 and 2.

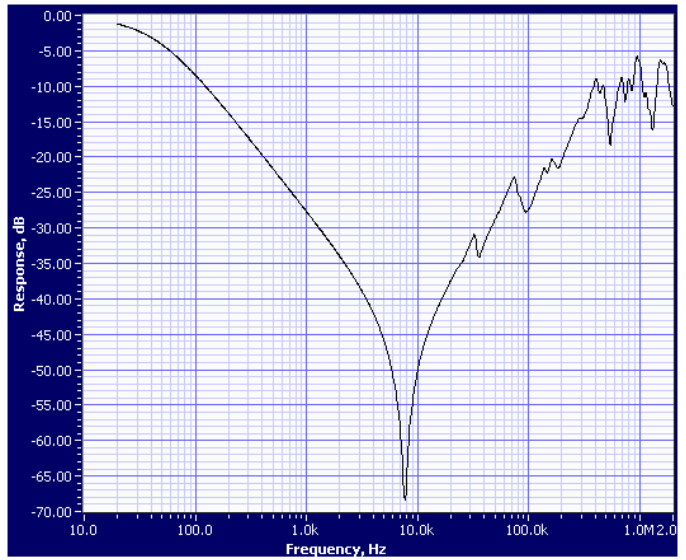

Fig. 4 FRA of HV winding with core removed

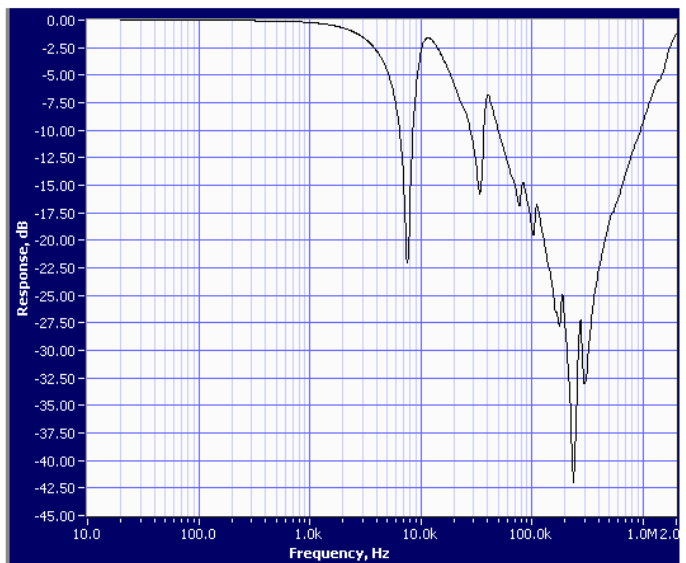

Fig. 5 FRA of LV winding with core removed

It can be seen from Figures 4 and 5 that at low frequency range, losses are much less than the previous case. This is because the magnetic circuit of the core determines the inductive characteristics in the low frequency range and since the core is removed and the winding inductance is small, the 
output voltage is almost equal the input voltage. At higher frequencies the distributed capacitances in the transformer will dominate and the frequency response will depict more resonant frequencies.

To determine the sensitivity of the FRA method to changes in winding inductance, the effect of changing tap position was examined. Figure 6 shows the FRA of the HV winding with the tapping winding open circuit. The effect is noticeable over the whole frequency range.

In the low frequency range the oscillation is most likely to be affected by coil configuration, in the middle range by layer and section effects and at higher frequencies by individual turns. Resonance points will appear only in the high frequency range. In the low frequency range, the out put voltage is zero because of the shorted turns and the core removal. At higher frequency range, effect of capacitances will be dominate and the output voltage will exist with many resonant points.

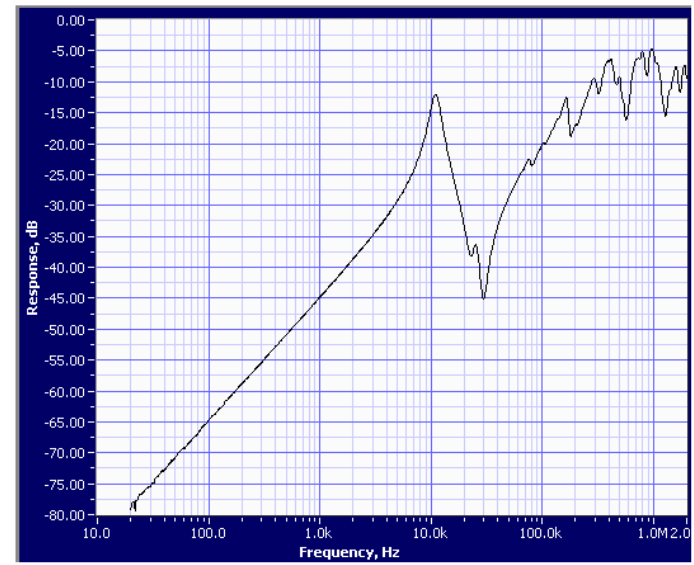

Fig. 6 FRA of HV winding with tapping winding open circuit

The effect is more noticeable in the low frequency range (less than $10 \mathrm{kHz}$ ) over the entire frequency range.

\section{TRANSFORMER MODEL}

The practical application of any diagnostic technique to detect mechanical damage in a transformer depends on its sensitivity to change in the distributed inductance and capacitance. Every transformer has a unique transfer function and can be modelled by a string of inductances to earth and shunted by their stray capacitances between windings. The equivalent circuit is useful in modelling the sensitivity of FRA to winding changes. It also can be used for the localization of partial discharges. A change in response could be related to a calculated amount of winding deformation. FRA results can be used to construct models of transformer winding. These models can be used to relate frequency response data to the transformer mechanical structure and to quantify significant winding changes. High frequency transformer models are based on lumped circuit approach where elements of transformer including windings, core, etc are represented by electrical parameters that can be measured or calculated. The selection of these parameters determines the accuracy of the model. The influence imposed by the core into the inductance calculations has been discussed by many researchers [6]. However, all the previous studies have studied this point theoretically. In this work, practical FRA is obtained for the transformer under study with and without the core. A computer model is proposed for each case. The frequency response of each model is compared with the corresponding practical one. In this case, effect of the core can be elaborated in a wide range of frequencies.

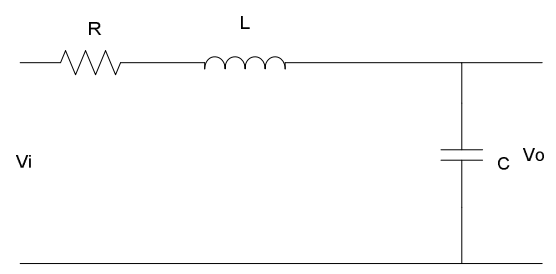

Fig. 7 Equivalent circuit of HV winding

Figure 7 shows the proposed model of the HV winding only (with core and $\mathrm{LV}$ winding removed). In the model, $\mathrm{R}$ is the total resistance of the winding, $\mathrm{L}$ is the total leakage inductance and $\mathrm{C}$ represents the winding capacitance to ground. The transfer function of the circuit shown in Figure 7 can be written as:

$$
\frac{V_{o}}{V_{i}}=\frac{\frac{1}{L C}}{S^{2}+\frac{R}{L} S+\frac{1}{L C}}
$$

Or in the frequency domain:

$$
\begin{aligned}
& \frac{V_{o}}{V_{i}}=\frac{\frac{1}{L C}}{\sqrt{\left(\frac{1}{L C}-\omega^{2}\right)^{2}+\left(\frac{R}{L}\right)^{2} \omega^{2}}} \angle-\tan ^{-1} \frac{\frac{R}{L} \omega}{\frac{1}{L C}-\omega^{2}} \\
& \Rightarrow\left|\frac{V_{o}}{V_{i}}\right|_{d B}=20 \log \frac{1}{L C}-10 \log \left(\left(\frac{1}{L C}-\omega^{2}\right)^{2}+\left(\frac{R}{L}\right)^{2} \omega^{2}\right)
\end{aligned}
$$

Hence, it is possible to plot the frequency response of the transfer function along with the frequency response of the practical test. In this way, a comparison of the two curves may be made and an assessment made of the degree of accuracy of the derived model.

Figure 8 shows a comparison between the frequency response obtained from the FRA test and the frequency response of the proposed model. The plot shows a difference between the experimental and model responses in the low frequency range, this may be attributed to the ignorance of the core effect. In the medium and high frequency range, the model response is not ideally accurate due to the complex shape of the measured frequency response, which depicts a number of resonant points. 


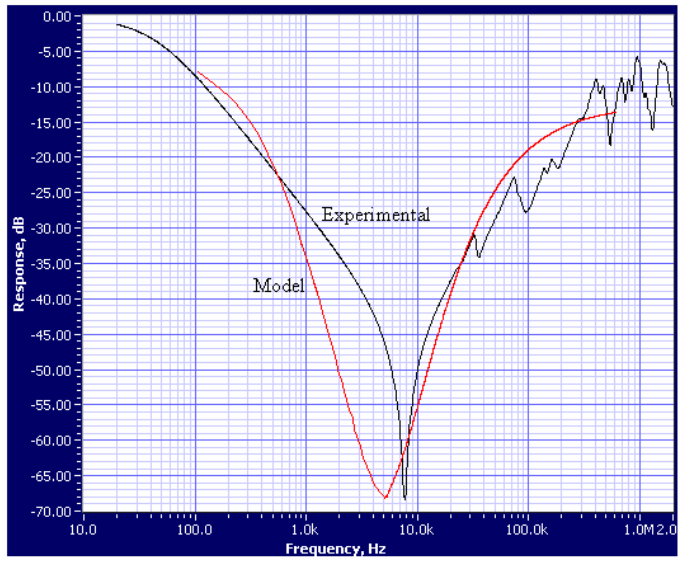

Fig. $8 \mathrm{HV}$ winding Experimental and model frequency responses

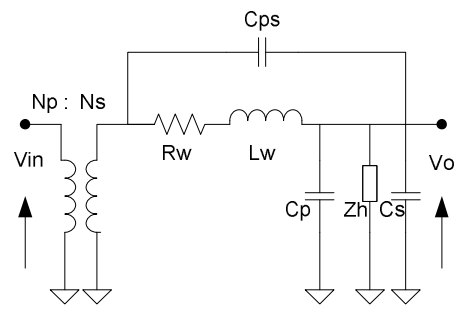

Fig. 9 Transformer equivalent circuit

Figure 9 shows the transformer general equivalent circuit proposed by Douglass[7]. In the model the following parameters are in their lumped form and all referred to the secondary side:

1) an ideal transformer for ratio purposes only.

2) $C_{p}$ and $C_{s}$ represent the primary and secondary winding capacitances to ground respectively.

3) $\mathrm{L}_{\mathrm{w}}$ is the equivalent leakage inductance of the primary and secondary windings.

4) $Z_{h}$ is the core exciting impedance (resistance and inductance in parallel).

Syed et al.[8] proposed three high frequency transformer equivalent circuits by dividing the general model into low, medium and high frequency range models, reflecting parameter significance. This paper presents a general high frequency transformer model that can be used for FRA.

The transfer function of the circuit shown in Fig. 9 can be written as:

$\frac{V_{o}}{V_{i}}=\frac{\frac{C_{p s}}{C_{e q}} s^{2}\left(s+\frac{R_{w}}{L_{w}}\right)}{s^{3}+s^{2}\left(\frac{R_{w}}{L_{w}}+\frac{1}{R_{h} C_{e q}}\right)+s \frac{1}{C_{e q}}\left(\frac{1}{L_{h}}+\frac{R_{w}}{L_{w} R_{h}}+\frac{1}{L_{w}}\right)+\frac{R_{w}}{L_{h} L_{w} C_{e q}}}$

Where $C_{e q}=C_{s}+C_{p}+C_{p s}$

The design data of the transformer is used to compute the inductances and capacitances shown in the above equation[9, 10].

It can be observed from the transfer function and its phase plot shown in Fig. 10 that at low frequencies, the influence of capacitance is negligible and the winding behaves as an inductor. This is due to the fact that flux penetration of the core is significant and hence $\mathrm{Z}_{\mathrm{h}}$, the core excitation impedance, is included. As the frequency increases, the circuit capacitances dominate.

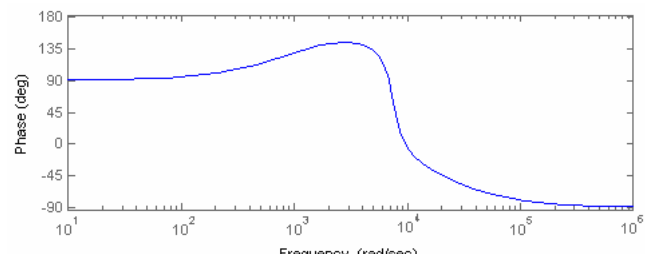

Fig. 10 Phase plot of the model TF

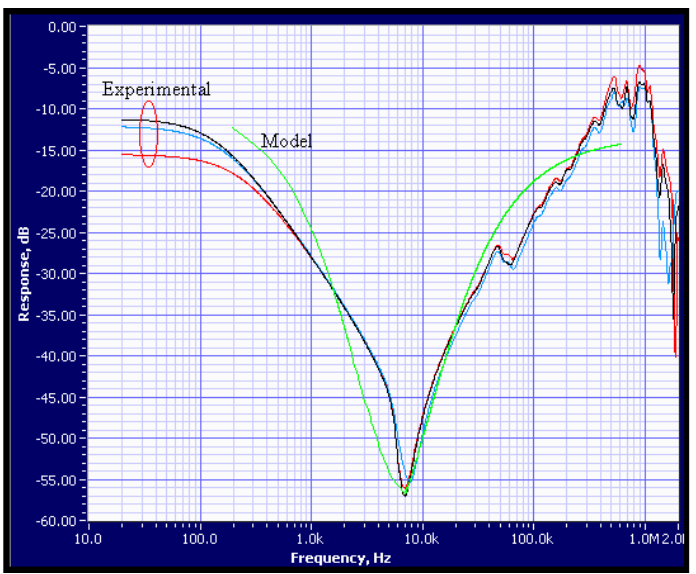

Fig. 11 Transformer actual and model frequency responses

Figure 11 shows the experimental and model frequency responses. At low frequency range, the experimental response tends to shift towards left. This may be attributed to the presence of residual magnetism in the core. The model can not simulate the distortion appears in the very high frequency range which is most probably caused by the end leads associated with tap-changer leads and bushing tails.

It can be seen from Fig. 11 that in the high frequency range, the model and experimental responses are correlated to far extent. It can be concluded that the transformer frequency response is better emulated by retaining the core exciting impedance.

\section{4. CONCLUSION}

Experimental results for FRA technique have been discussed in this paper. Effect of bushings, core, oil and tank on FRA has been elaborated. Sensitivity of the FRA method to change of tap position was examined. The main problem about FRA techniques is to interpret the observed evolution of the frequency response in order to identify failures. The paper presents a Transformer high frequency computer modeling that can be used with the FRA. The physical meaning of the model parameters allows the identification of the problem inside the 
transformer. Results show that transformer frequency response is better emulated by retaining the core exciting impedance. The proposed model is easy to implement and used as a successful tool for FRA and can be used for condition monitoring of power transformer.

\section{ACKNOWLEDGEMENT}

The authors would like to thank the Cooperative Research Centre for Integrated Engineering Asset Management (CIEAM) for funding this research. The authors greatly acknowledge the co-operation of Mr. Karl Haubner (Doble) and Mr. Wayne Proud (Western Power) in the experimental tests.

\section{REFERENCES}

[1] T. K. Saha, "Review of Modern Diagnostic techniques for Assessing Insulation Condition Aged Transformers," IEEE Transaction on Dielectrics and Electrical Insulation, vol. 10, pp. 903-917, 2003.

[2] L. Coffeen, J. Britton, and J. Rickmann, "A new technique to detect winding displacements in power transformers using frequency response analysis," 2003, p. 7 pp. Vol.2.

[3] P. M. Nirgude, B. Gunasekaran, Channakeshava, A. D. Rajkumar, and B. P. Singh, "Frequency response analysis approach for condition monitoring of transformer," 2004 Annual Conference on Electrical Insulation and Dielectric Phenomena, pp. 186-189.

[4] K. Jong-Wook, P. ByungKoo, J. Seung Cheol, K. Sang Woo, and P. PooGyeon, "Fault diagnosis of a power transformer using an improved frequency-response analysis," Power Delivery, IEEE Transactions on, vol. 20, pp. 169-178, 2005.

[5] M. Arshad, "Remnant Life Estimation Model Using Fuzzy Logic for Power Transformer Asset Management," PhD thesis, Curtin University of Technology, 2005.

[6] K. G. N. B. Abeywickrama, A. D. Podoltsev, Y. V. Serdyuk, and S. M. Gubanski, "Computation of Parameters of Power Transformer Windings for Use in Frequency Response Analysis," Magnetics, IEEE Transactions on, vol. 43, pp. 1983-1990, 2007.

[7] D. A. Douglass, "Potential Transformer Accurcy at $60 \mathrm{~Hz}$ Voltages above and Below rating and at Frequencies above $60 \mathrm{~Hz}, "$ IEEE Transaction on Power Apparatus and Systems, vol. PAS-100, No 3, 1981.

[8] S. M. Islam, K. M. Coates, and G. Ledwich, "Identification of High Frequency Transformer Equivalent Circuit Using Matlab from Frequency Domain Data," IEEE Industry Applications Socity Annual Meeting, pp. 357-364, 1997.

[9] BHEL Transformer Handbook. New Delhi: Tata McGraw Hill Publishing Company Limited.

[10] G. F.W., Inductance calculations. Newyork: Dover puplications Inc. 